\title{
Das Mittelwürm in Mitteleuropa und angrenzenden Gebieten
}

\author{
Von Hugo Gross, Bamberg \\ Mit 1 Abbildung im Text
}

$\mathrm{Z}$ us a mmenfassung: Das mittelwürmzeitliche „Interstadial W I/II“ spielt besonders in der urgeschichtlichen Literatur seit 1931 im Gegensatz zu der quartärgeologischen eine große Rolle. Zahlreiche mit der $\mathrm{Cl}^{14}$-Methode bzw. Sedimentanalyse datierte Schichten verschiedenartiger spätpleistozäner Ablagerungen sind im Mittelwürm zwischen ca. 50000 und 30000 B.P. fast durchweg interstadial; in ca. 10 terrassenmorphologisch datierten Würm-Lößpaketen kommt eine mittelwürmzeitliche und eine ganz sd,wache spätwürmzeitliche Verlehmungszone vor. Diese Fakten beweisen die Realität eines interpleniglazialen Mittelwürms zwischen dem ersten und dem zweiten Kältemaximum im Altwürm und Jungwürm. Das Klima im Mittelwürm war im Durchschnitt ziemlich kühl mit wärmeren und kälteren Abschnitten. Das horizontale Wachstum des Würm-Eises in den Alpen und des Weichseleises im Norden war in dieser Zeit unterbrochen, das Inlandeis stagnierte, die Eisfronten oszillierten mehr oder weniger weit. Am Ende des Mittelwürms floß das Würm-Eis schließlich über die nördlichen Alpenpässe ins Vorland und das Weichsel-Eis im Norden aus dem Ostsee-Becken heraus, beide bis zu ihren äußeren Jungendmoränen (Jungwürm). In Mitteleuropa scheidet das Mittelwürm das Mittel- und Jungpaläolithikum. Ins Mittelwürm ist bisher vielfach die Verlehmungszone in Löß bei Görtweig (Niederösterreich) als bekannteste Bildung dieser Zeit gestellt worden; mit ihr ist im dreifachen Stillfried A-Komplex fossiler Böden im Löß sehr arider Gebiete (östl. Niederösterreich und ČSSR) die basale Verlehmungszone parallelisiert worden. In den letzten Jahren haben verschiedene Untersuchungsmethoden in Unter-Wisternitz und 1963 in Niederösterreich palynologische $\mathrm{d}$ i e s e Verlehmungszone als letztinterglazial (R/W) festgestellt. Für die ebenfalls nicht terrassenmorphologisch datierbare namengebende einfache Verlehmungszone bei Göttweig ist das noch nicht nachgewiesen. Unter diesen Umständen muß für das Mittelwürm der Name "Göttweiger Interstadial" (F. E. ZEUNER) durch einen anderen ersetzt werden, etwa "Würm-Interpleniglazial“.

S u mmary: The Mid-Würmian "interstadial W I/II" plays a prominent part in the literature dealing with Pleistocene archaeology since 1931. This is not the case in the geological literature. Numerous strata dated by $\mathrm{Cl}^{14}$ measurement and sedimentanalysis, respectively, to be of MidWürmian age (between ca. 50000 and 30000 B.P.) in various Upper Pleistocene sequences are for the most part interstadial; ca, ten Würmian loess sequences dated by terrace morphology contain a Mid-Würmian considerable loam zone and a very weak younger loam zone. These facts prove the reality of an interpleniglazial Mid-Würm between the two cold peaks of Early Würm and Young Würm. The interpleniglacial climate of Middle Würm was on the average rather a cool temperate one interrupted by warmer and colder oscillations. Within the Alps and in northern Europe the expansion of the Würmian and Weichselian ice-masses was stopped, they stagnated, their fronts oscillated more or less widely. At the close of the Middle Würm, the Würmian ice overflowed the northern Alpine passes and the Weichselian ice the Baltic basin, both as far as the Young Endmoraine girdle (Young Würm). In Central Europe Middle Würm divides the Middle and Upper Palaeolithic periods. To the Middle Würm has often been ascribed heretofore the well known loam stratum within the loess of Göttweig (Lower Austria), and with this loam has been correlated the basal loam zone of the triple Stillfried A complex of fossil soils displayed by loess sections of particularly arid regions (ČSSR and eastern Lower Austria). Recent studies (the latest by palynology) of $t h$ is loam have proved it to be of Riß, Würm Interglacial age. Till now, this correlation has not yet been demonstrated for the loam stratum of the type locality of Göttweig (also not datable by terrace morphology!). Therefore the te r m "Göttweig Interstadial" must be replaced by another name: Würmian Interpleniglacial

Der jahrzehntelange Streit um die Geochronologie des Spätpleistozäns (vom Ende des Riß bis zum Ende von Würm, ca, 100000 Jahre) dreht sich um die Frage, ob die Letzte Eiszeit ungefähr in ihrer Mitte durch ein bedeutendes Interstadial unterbrochen war (Soengel 1919) oder nicht (PENCK 1922). Die allermeisten mitteleuropäischen Quartärgeologen sind Penck gefolgt, die große Mehrzahl der Urgeschichtler aber hat die LößChronologie von SOERGEL angenommen, weil ihre paläofaunistischen, paläofloristischen, archäologischen und (seit 1941) sedimentanalytischen Befunde von Ausgrabungen besonders in Höhlen und Abris für die Richtigkeit der paläoklimatologisch begründeten Gliede- 
rung der Letzten Eiszeit durch Soengel in W I (Früh- oder Altwürm), W I/II („Hauptschwankung“ oder "großes Würm-Interstadial“, nach Soengel repräsentiert im Löß durch die Verlehmungszone von Göttweig bei Krems a. d. Donau) und W II a + b (Jungwürm) sprechen. Mit Hilfe der $\mathrm{C}^{14}-$ Methode ist es, wenn genügend $\mathrm{C}^{14}-\mathrm{Daten}$ vorliegen, möglich, Schichten von gleichem paläoklimatischem Aussagewert in den verschiedenartigen spätpleistozänen Ablagerungen im Periglazial und in den Moränengebieten mit Berücksichtigung der Stratigraphie (also der relativen Datierung) zu konnektieren und so eine Geochronologie des Spätpleistozäns in großen Zügen aufzustellen.

Mit seinem "Würm I" hat Soergel natürlich ein Frühw ü rm und nicht ein Spät$s$ a a le (Warthe) gemeint, obwohl er sein W I mit Warthe parallelisiert hat, aber damals wurden die Warthe-Moränen von der Preuß. Geolog. Landesanstalt amtlich dem W e i c h s e 1 - Maximum zugewiesen.

Als B e ginn des Mitte $1 \mathrm{w}$ ü $\mathrm{rms}$ ist im Moränengebiet das Ende des maximalen Altwürm-Stadials, des dritten, also nach dem zweiten Interstadial, zu nehmen; es ist das erste Kältemaximum der Letzten Eiszeit. Im Weichsel-Vereisungsgebiet wird dieses Stadial repräsentiert durch den Vorstoß des (Main) Irish Sea-Gletschers in NW-England zwischen ca. 56000 B.P. (=Ende des 2. Altwürm-Interstadials von Chelford-BrörupLoopstedt kurz vor dem „kalten“ Moustérien von Lebenstedt bei Braunschweig, Gro-2083: $55000 \pm 1000$ B.P., von dem ich die Pollenspektra von SELle gesehen habe) und ca. 42000 B.P. (C $14-D a t e n$ von Upton Warren in Worcestershire), als sich der genannte Gletscher für viele Jahrtausende zurückzuziehen begann (Coope, Shotron \& Strachan 1961), verspätet durch die abkühlende Wirkung der nur ca. $32 \mathrm{~km}$ entfernten Inlandeisfront. Weiter südlich hat sich nämlich diese Klimabesserung merklich früher ausgewirkt, sie ließ die begrabene Moorbildung (1,60 m mächtig, mit Gyttja, die nicht im Tundrenklima gebildet wird) in Moershoofd in Holland spätestens um 47000 B.P. entstehen (ZAGwiJN 1961). Im Würm-Vereisungsgebiet bewirkte das dritte Stadial, das auf die Bildung der Großweiler Schieferkohlenschicht der Pollenzone 13 von H. REICH (1953) folgte, die stärkste Schüttung von Altwürm-Vorrückungs-Schotter der maximalen Altwürm-Gletscher, die in den Alpen oszillierten, durch die Alpenfluß-Täler ins nördliche Alpenvorland ("unterer Glacialschotter" von Penck 1882, insgesamt bis ca. 50 m, z. B. Murnauer und Laufenschotter).

Das Ende des Mittelwürms ist gekennzeichnet durch das Aufhören der letzten Begehung hochliegender Höhlen (bis $1200-2000 \mathrm{~m}$ ) der randlichen Alpen durch Höhlenbären und die ersten Jungpaläolithiker, was für ein recht mildes Klima spricht. Diese Jungpaläolithiker waren Träger des Olschewien (=östliche Fazies des ältesten Aurignacien), das keine oder nur wenige primitive Steingeräte in den Bärenhöhlen hinterlassen hat und durch die Potočka- oder Lautscher Knochenspitzen nebst durchbohrten Tierzähnen gekennzeichnet ist; sie liegen in den oberen Schichten der letzten Anhäufung großer Mengen von Höhlenbärenresten. Das Olschewien der Potočka-Höhle (1700 m hoch in den Ostkarawanken) dürfte ungefähr ebenso alt sein wie in der Istállóskö (Stall)-Höhle in Ungarn, nämlich ca. $30710 \pm 600$ (Gro-1935) Cl4-Jahre. Das gleiche dürfte für die Lautscher Knochenspitze der Bären- oder Tischofer-Höhle bei Kufstein (s. unten) und für die letzte Begehung des Liegl-Lochs bei Tauplitz in der Steiermark gelten. Aus der in $2008 \mathrm{~m}$ hoch im Toten Gebirge bei Bad Aussee liegenden Salzofen-Höhle lieferte Holzkohle aus der Hauptfundschicht der Höhlenbärenreste, die nach der sedimentanalytischen Untersuchung von E. ScHMID (1958) aus dem "großen Würm-Interstadial W I/II “ stammt, das C14-Datum Gro-761: $34000 \pm 3000$ B.P. Ungefähr um 31000 B.P. begann, wie besonders in Südfrankreich festgestellt worden ist (Movius 1960) eine allgemeine starke Abkühlung, die in den Alpen das Wachstum der Würm-Eismassen wieder aktivierte, bis es um 30000 B.P. über die nördlichen Alpenpässe zu fließen begann (Beginn der Alpenvorland-Vergletscherung $=$ Jungwürm). Im Gebiet der Weichselvereisung ist dieser Zeit- 
punkt noch nicht mit der $\mathrm{C}^{14}$-Methode datiert worden, er dürfte aber ungefähr die gleiche Zeitstellung haben wie im Würm-Vereisungsgebiet. In NO-Wales verschloß der letzte Vorstoß des Irish Sea-Gletschers zwei Höhlen mit Spät-Aurignacien (also im Jungwürm) durch seine Grundmoräne (PEAKE 1961). Im Jungwürm lag (von ca. 25000-17 000 B.P.) das zweite (absolute) Kältemaximum der letzten Eiszeit.

Das Mittelwürm (Gross 1958, S. 182) zwischen ca. 50000 und 30000 B.P. (abgerundet) ist also interpleniglazial; es ist schon seiner Länge wegen natürlich nicht ein Interstadial im üblichen Sinne (Klimaabfolge: „arktisch“ — „subarktisch“ - kühl-temperiert [boreal] — „subarktisch“ - „arktisch“), sondern ein im Durchschnitt ziemlich kühles Interstadial mit kälteren und wärmeren Oszillationen, wie aus der Fossilführung von ca. 30 (nach Weglassung der unsicher gewordenen) durch $\mathrm{C}^{14}$-Messung datierten Schichten hervorgeht. Dazu kommen noch mittelwürmzeitliche Verlehmungszonen in ca. 12 terrassenmorphologisch oder anders von den zuständigen Feldgeologen datierten Würmlöß-Paketen. Wir wissen also über das Mittelwürm mehr als über das Alt- und Jungwürm. Einige besonders wichtige Mittelwürm-Bildungen aus verschiedenartigen spätpleistozänen $\mathrm{Ab}$ lagerungen beweisen das.

\section{Mittelwürm-Bildungen in den Vereisungsgebieten}

1. In NW-England hat nach Coope, Shotton \& Strachan der (Main) Irish Sea-Gletscher, der als erster nach dem Eem dieses Gebiet erreichte, bei seinem Abrücken für viele Jahrtausende seit ca. 42000 B.P. durch seine Schmelzwässer relativ rasch die ca. $9 \mathrm{~m}$ mächtige Salwarpe-Flußterrasse mit mehreren dünnen, aber fossilreichen Teichböden ca. $30 \mathrm{~km}$ südlich von der Inlandeisfront aufgeschottert. Die beiden Pollenspektra enthalten über 90\% Nichtbaumpollen (maximal 99,5\%!), beweisen hier aber nicht, wie bisher anderwärts allgemein angenommen worden ist, ein pleniglaziales Tundrenklima; denn die chorologische Analyse der in Upton Warren nachgewiesenen rund 80 Pflanzen- und $170 \mathrm{Käfer-Arten} \mathrm{ergab} \mathrm{eine} \mathrm{Mischung} \mathrm{von} \mathrm{nordischen} \mathrm{und} \mathrm{südlichen} \mathrm{Arten,} \mathrm{die} \mathrm{für} \mathrm{ein}$ Klima "more akin to that of southern Sweden at the present day" spricht. Die interstadiale Erwärmung muß also schon geraume Zeit vor 42000 B.P. begonnen haben.

2. Die Bären-oder Tischofer-Höhle bei Kufstein (Nordt i r o l). Diese relativ kleine Bärenhöhle liegt im Kaisertal des Kaisergebirges in ca. $600 \mathrm{~m}$ Meereshöhe im Einflußbereich des großen würmzeitlichen Inn-Gletschers nicht weit von seinem Austritt aus den Alpen ins bayerische Alpenvorland $80 \mathrm{~m}$ über dem Bett des Kaiserbaches; sie wurde 1906 unter der Leitung des bekannten Münchener Paläzoologen ScHLOSSER ausgegraben, der 1909 (1910) ausführlich über die geologischen, paläofaunistischen, archäologischen und paläanthropologischen Ergebnisse berichtete. Danach war die Höhle vom Würmeis im Jung w ü $\mathrm{rm}$ verschlossen und begraben. Ihre Höhlenbärenschicht mit einer (in der Istállóskö-Höhle in Ungarn ca. $31000 \mathrm{C}^{14}$-Jahre alten) charakteristischen Lautscher Knochenspitze (Zотz 1951, S. 201, 202) kann nicht in der kühleren zweiten Hälfte des Riß/Würm-Interglazials abgelagert worden sein, wie ScHLosser (1. c.) angenommen hat, sondern in der im wesentlichen interstadialen Zeit zwischen dem Jungwürm und der altwürmzeitlichen Ausfüllung des Kaisertals und des nahen Inntals, also im Mittelwürm (vgl. Gross 1964).

\section{Mittelwürm-interstadiale Schieferkohlen im Alpenvorland}

In und auf der schiefen Ebene des Schotters aus dem Altwürm und Mittelwürm haben die Schmelzwässer nur selten eine Moorbildung $\mathrm{n}$ i $\mathrm{ch}$ t verhindern können. Eine solche Ausnahme ist das von E. EBERs (1960) in einem Drumlin des würmeiszeitlichen Inn-Gletschers bei Hörmating (Kreis Bad Aibling) beschriebene und stratigraphisch eingestufte kleine begrabene Moor, das A. Michelen entdeckt hat; ich habe den Aufschiluß 1959 zweimal zur Probeentnahme besucht. Der sehr stark zersetzte Carex-Hypnum-Sumpftorf 
war wie die stark sandige Seekreide im Liegenden völlig pollenfrei, zweifellos infolge wiederholter Austrocknung und Durchnässung; nur die dünne tonige Zwischenschicht enthielt Pollen von Holzarten, die bis in die Subarktis hinein gehen. Der Torf lieferte das C14-Datum Gro-2593: $45300 \pm 1000$ B.P., ein nach längerem Suchen gefundener Fichtenast darin (Gro-2595), dessen Ausformung nach Mitteilung der Sammlerin auf einen weiten Wassertransport aus einem am Alpenrande ca. $100 \mathrm{~m}$ höher liegenden von Schmelzwässern aufgearbeiteten letztinterglazialen oder eher altwürmzeitlichen interstadialen Schieferkohlenflöz schließen läßt, ist älter als $53000 \mathrm{C}^{14}$-Jahre. Selbst wenn dieser Fichtenast autochthon wäre, könnte der Hörmatinger Torf, falls er durch jüngere organogene Substanzen verjüngt wäre, nur aus dem zweiten Altwürm-Interstadial stammen, wogegen aber die Mächtigkeit des liegenden Basalschotters (mehr als $15 \mathrm{~m}$ !) spricht. Ganz abwegig ist im Hinblick auf den dürftigen Fossilinhalt die Einstufung des Hörmatinger begrabenen Moors in das Riß/Würm-Interglazial. Eine fossilführende Ablagerung zwischen zwei kaltzeitlichen Schichten ist nur dann interglazial, wenn ihr Pollendiagrammoder/und ihr sonstiger Fossilinhalt für ein so langes und so warmes Klima sprechen, daß damals auch Fennoskandinavien inlandeisfrei gewesen sein $\mathrm{mu}$ ß. Diese Schlußfolgerung ist nach meiner pollenanalytischen Untersuchung der von Frau Dr. E. Ebers 1959 in Zeifen (Kreis Laufen) im Salzach-Gletschergebiet unter Laufenschotter entdeckten bis $4 \mathrm{~m}$ mächtigen außerordentlich pollenreichen Kalkmudde zwingend, im Vergleich damit unter keinen Umständen im Falle Hörmating; daher kann dort auch nicht von „interglazialer Braunerde" die Rede sein.

Das C ${ }^{14}-D a t u m ~ B-20: 29000 \pm 1500$ B.P. der von P. BECK (1938) gesammelten Schieferkohle seiner "S piezer Schwankung " ist wahrscheinlich etwas verjüngt, ebenso das C14-Datum (rund 29000 B.P.) des Karrestobel-Torfes $\mathrm{N}$ von Ravensburg (Württemberg), der nach PENCK \& BRüCKNER (1909, S. 422) in der Laufen-Schwankung zwischen den beiden Haupt-Endmoränenzügen Oberschwabens entstanden sein dürfte (dieser Torf ist so außerordentlich pollenarm, daß bisher niemand ein Pollenspektrum für ihn ermitteln konnte).

\section{Mittelwürm-Bildungen im Periglazial außerhalb der Höhlen- und Löß-Gebiete}

1. Das von DE Vries (1958 b, Tafel 52, 3) und Z ZaGwiJN (1961, Fig. 7) abgebildete und untersuchte Profil von Amersfoort in Holland reicht vom marinen Eem bis ins Postglazial; mittelwürmzeitlich ist nach dem C14-Datum Gro-1259 und 1276: $34730 \pm 700$ bzw. \pm 800 B.P. ein (nachträglich) sehr stark kryoturbierter sehr humoser Lehm, der nach dem Pollenspektrum als pleniglazial bezeichnet wird. Andere holländische Profile ( $Z_{\text {AgwijN }}$ 1961, Appendix 5) enthalten mittelwürmzeitliche Schichten, die sicher interstadial sind: Hengelo (Torf und Gyttja) Gro-1763: $37400 \pm 800$ B.P., Moershoofd I (BraunmoosTorf, $20 \mathrm{~cm}$ mächtig) Gro-1852: $38000 \pm 1000$ B.P., Moershoofd II + III (1,60 m Braunmoostorf und Gyttja) Gro-1715: $44500 \pm 1500$ B.P. bzw. Gro-1718: $46500 \pm 2000$ B.P. (mindestens!). Nach Andersen, DE Vries \& ZaGWijn (1960) dürfte der Torf von Breda (P 25a) Gro-2007: $31750 \pm 400$ B.P. aus einer kühl-temperierten (also interstadialen) Waldzeit stammen.

2. In Polen stellte das Ehepaar J. DyliK und A. Dylikowa (1961) in Toteislöchern der Grundmoräne des Warthe-Stadials im Gebiet von Łódž im Hangenden von eemzeitlichen und altwürminterstadialen Moorschichten einen erheblich mächtigeren minerogenen Wanderschutt fest; in ihm konnten in zwei Horizonten die starken Frostwirkungen zweier Kältemaxima der Letzten Eiszeit (sicher vom Altwürm- und Jungwürm-Maximum) und dazwischen die Reste eines zerstörten Bodens (sicher aus dem Mittelwürm) festgestellt werden. Für eine Torfbildung war hier also das Klima in dieser Zeit noch nicht humide genug. 


\section{Mittelwürm-Bildungen in den Sedimenten von Höhlen und Abris}

Mit Hilfe der Methode von LAIs (SCHMID 1958, VÉRtes 1959) konnte in spätpleistozänen Sedimenten durch die Sedimentanalyse mit Berücksichtigung der paläofaunistischen, paläofloristischen (Vorsicht bei der Auswertung von Holzkohle-Bestimmungen!) und archäologischen Befunde (Beispiel: Weinberghöhlen-Monographie von Zotz u. Mitarb. 1955) das Mittelwürm als Periode mit unbeständigem interstadialem Klima festgestellt werden. Die Bedeutung dieser langen Periode für die Urgeschichte hat Véntes $(1955,1958)$ ausführlich erläutert; man muß aber in diesen Arbeiten die Datierung mit Hilfe der Sonnenstrahlungskurve im Sinne von KöPPEN-WEGENER-SOERgEL ausklammern.

1. Istállóskö (Stall)-Höhle im Bükkgebirge (535 m ü.d.M.) in Ungarn (VÉRTEs 1955): Holzkohle aus dem oberen Teil des Abschnitts „W I/II“ lieferte nach Säure-Alkali-SäureVorbehandlung für das Aurignacien II (VÉrTEs) = Olschewien II das zuverlässige C $\mathrm{C}^{14}$ Datum GrN-1935: $30900 \pm 600$ B.P. und für das Aurignacien I (VÉrtes)=Olschewien I, von der oberen Kulturschicht durch eine $1 \mathrm{~m}$ mächtige sterile Schuttschicht getrennt, das

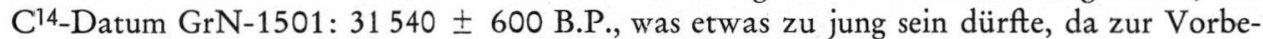
handlung wegen der Kleinheit der Holzkohle-Probe kein Alkali angewandt werden konnte.

2. Höhle Pod hradem im Mährischen Karst: im oberen Teil von „W I/II" Holzkohle Gro-848: $32990 \pm 1500$ B.P. und Humus Gro-1724: $32420 \pm 470$ B.P. (VAloch 1961, S. 448).

3. Grotte du Renne bei Arcy-sur-Cure (Yonne): Périgordien I (=Châtelperronien): Gro-1736: $33500 \pm 400$ B.P. und Gro-1742: $33640 \pm 250$ B.P., nach der Begleitfauna Klima interstadial (Movius 1960, S. 366).

4. Nietoperzowa (Fledermaus)-Höhle von Jerzmanowice bei Krakau (Zotz 1951, S. 164-166, Freund 1952): eine Humusschicht etwas höher als eine interstadiale Schicht mit Wald- und Steppentieren lieferte für die dortige reiche (Feuerstein-) Blattspitzen-Industrie das Cl4-Datum Gro-2181: $38160 \pm 1250$ B.P. (W. ChMielewsKi 1961).

A. Leroi-Gourhan (1960) gibt auf Grund pollenanalytischer Untersuchungen von Höhlensedimenten mit archäologischen, paläontologischen und $\mathrm{C}^{14}$-Datierungen in Frankreich für das Mittelwürm an: ca. $47000-42000$ B.P. „Interstade de Göttweig(?)“, um 38000 B.P. kalte Oszillation, ca. $37500-34000$ B.P. interstadiales Klima im Châtelperronien, ca. $34000-31000$ B.P. kalte Periode in der Dordogne (im südöstlichen Mitteleuropa aber interstadial).

\section{Mittelwürm-Bildungen in Löß-Profilen}

Löß-Profile sind wohl die tückischsten geologischen Profile, da infolge der Feinkörnigkeit des losen Lösses Umlagerungen und Abtragungen durch Winderosion und wegen der vorherrschenden Hanglage auch durch Hangabspülung und im Eiszeitklima auch durch Solifluktion möglich waren, so daß Lößprofile recht oft lückenhaft sein können. Da sogar im klassischen Land der Lößforschung Ớsterreich, aber auch in Süddeutschland die fossilen Böden planmäßig höchstens durch die Ermittlung der Kalk- und Humus-Kurce, sonst aber nur makroskopis ch beurteilt worden sind, ist der Quartärgeologe auf andere leider nur recht selten anwendbare zuverlässigere Methoden für die Datierung der fossilen Böden und damit für die paläoklimatische Interpretation angewiesen (Datierung von Würm-Lößpaketen durch die Feststellung des Alters ihrer Unterlage seitens der zuständigen Feldgeologen, in der Regel mit Hilfe der Terrassenmorphologie); dabei ist zu beachten, daß der sehr schwache Paudorfer Boden ("W II/III“ oder "W II a/b“) oft sehr undeutlich sein oder gar fehlen kann.

$\mathrm{Da}$ auf der jüngsten Niederterrasse (NT II von Jungwürm) niemals in primärer Lagerung (Jungwürm-) Löß vorkommt, müssen wir annehmen, daß eine NT mit Lößdecke 
(ohne fossilen Boden) eine ältere NT I ist; das beweist nach den Untersuchungen von WERnERT bei Straßburg (1957) die Terrassentreppe von Schiltigheim (Riß II-Terrasse) Lingolsheim (NT I) - Roettig (NT II). Der Löß eines bestimmten Stadials konnte nur auf eine Bildung eines älteren Stadials der gleichen Vereisung oder a uf noch ältere Bildungen fallen. Da für die vorletzte Vereisung (Riß, Saale) Befunde vorliegen, die für (mindestens) 2 Phasen (Riß I = Saale I oder Drenthe und Riß II = Spät- oder Jungriß und Saale II = Warthe) sprechen, müssen auch 2 Hochterrassen (HT)-Stufen vorkommen; das ist auch vielfach nachgewiesen. Auf einer HT kann also nur dann Riß-Löß vorkommen, wenn die HTStufe R I und der Löß R II-Löß ist. Wer für jede Eiszeit nur e i n e Terrasse und e in e n (ungegliederten) Schotterkörper annimmt, kann damit allein nicht beweisen, daß die Göttweiger Verlehmungszone letztinterglazial (R/W) ist: ein letztinterglazialer fossiler Boden im Lößprofil muß durch Verlehmung von Riß-Löß im R/W entstanden sein; Riß-Löß kann aber nicht auf die einzige HT-Stufe von Riß gefallen sein, sondern nur auf ältere Bildungen, es sei denn, daß die einzige H'T-Stufe von Riß I stammt; das läßt sich aber in Niederösterreich nicht beweisen. Ein paar Beispiele sollen zeigen, daß die Sache nicht so einfach ist, wie manche Quartärgeologen anscheinend annehmen. Als wichtiger chronologischer Leithorizont für die Löß-Chronologie darf das $C^{14}$-Datum (gemessen an Holzkohle) für das Aurignacien von Willendorf II, 4 (an der Donau bei Krems, N.-OO.) benutzt werden: Gro-1273: $31840 \pm 250$ B.P. und H 245-1276: $31700 \pm 500$ B.P., also beide identisch und auch deswegen zuverlässig, weil in Heidelberg $(\mathrm{H})$ für die chemische Vorbehandlung auch Alkali benutzt worden ist und das abgerundete Datum 32000 B.P. gut zu den übrigen C14-Daten für das ältere Aurignacien paßt (vgl. Movius 1960). Die Begleit-Flora und -Fauna dieser Kulturschicht in Willendorf sind nach A. FIETz und E. Thenius (Willendorf-Monographie von F. Felgenhauer in Mitteil. Prähist. Komm. Ósterr. Akad. d. Wiss. 8/9, 1956-1959) entschieden interstadial. Die autochthone Kulturschicht II, 4 liegt nach F. BRAndtner (in der gleichen Monographie S. 187, 196) auf einem mit Verlehmungsmaterial (aus W I/II, S. 184) vermengten Fließlöß (richtiger wohl: Schwemmlöß) a uf der altwürmzeitlichen Niederterrasse (NT I).

1. Lößprofil der Ziegelei Murg bei Säckingen im sehr ariden Oberrhein-Tal (Zotz 1951, S. 56-59, Guenther 1963): auf verwitterter Rißmoräne fand Guenther eine Moorbildung, die nach dem pollenanalytischen Befund (von Dr. TIDELSKI) aus dem späten R/WInterglazial und dem Übergang zum Altwürm (der allgemein sehr humide war) stammt; darauf liegt ein Würm I-Löß, von dem der obere Teil mit der Hauptmenge der LevalloisienArtefakte ungewöhnlich tief verlehmt ist, im hangenden Löß liegt ein viel schwächeres unzusammenhängendes Lehmband.

2. In der Ziegelei von Heitersheim bei Müllheim ebenfalls im Oberrhein-Tal (GuENTHER 1962/1963, ergänzt durch briefl. Mitteil.) liegt ein $28 \mathrm{~m}$ hohes Lößprofil auf Schwarzwald-Schottern, die nach Dr. L. ERB rißeiszeitlich sind, mit einer $1,50 \mathrm{~m}$ mächtigen Grobsand-Decke (R I/II-Interstadial?); im Lößprofil stellte Guenther folgende fossile Böden fest: a. rotbrauner ca. 2,50 m mächtiger Kremser Boden aus R/W über Lößkindel-Horizont mit über 0,75 m langen Kalkkonkretionen (typisch für den Älteren Löß nach SoergeL) = Riegel C, b. $2 \mathrm{~m}$ mächtiger nicht so intensiv gefärbter und daher schwächer verwitterter Riegel D-Boden auf Lößkindel-Horizont mit nicht einmal faustgroßen Kalkkonkretionen (typisch für den würmeiszeitlichen Jüngeren Löß nach SoERGEL), c. 2,50 m mächtiger „Göttweiger" (Riegel E) Boden, Färbung intensiver als bei RiegelD, aber nicht so intensiv wie bei Riegel C (Krems), auf Lößkindel-Horizont mit etwa faustgroßen Konkretionen, d. eine makroskopisch nicht sichtbare, aber durch die Kurven des Kalk- und Humusgehalts nachweisbare sehr schwache Bodenbildung (wohl Paudorf). Im Altwürm-Löß zwischen den Böden $a$ und $b$ hat GUENTHER mitunter eine weitere, aber schwächere Verlehmungszone gefunden; beide fossilen Böden im Altwürm-Löß zwischen den Böden a und c stammen 
sehr wahrscheinlich aus den beiden Altwürm-Interstadialen, von denen das zweite (BrörupLoopstedt) temperierter und bedeutend länger als das erste (Amersfoort (XII) war.

3. Thüringische Lößprofile (UNGer \& RAU 1961): am Rand des Trockenbeckens fanden UNGer \& RAU in der Ziegelei Kahla auf der oberen HT-Stufe (von Saale I = Drenthe) ein Lößpaket mit der starken letztinterglazialen Bodenbildung (Eem, Saale/Weichsel), darauf letzteiszeitlichen Löß mit einer etwas schwächeren Bodenbildung aus „W I/II" und einer oberen durch Vergleyung undeutlich gemachten aus „W II/III“, d. h. auf der Terrasse von Saale I = Drenthe akkumulierte zuerst Löß von Saale II = Warthe, dessen obere Schicht zur letztinterglazialen Bodenbildung verwitterte. In der Ziegelei KönNER im Trockenbecken stellten UNGER \& RAU auf der unteren HT von Saale II $=$ Warthe eine fluviatile fossilführende eemzeitliche Ablagerung fest, auf der ein letzteiszeitlicher Löß wie bei Kahla liegt.

4. Lößprofile im Regensburger Trockengebiet an der Donau (BRunnacker 1957): in der Ziegelei Köfering fand Brunnacker auf der oberen HT von Altriß = R I ein Lößpaket, wie UNGER \& RAU es in Kahla auf der oberen Terrasse von Saale I festgestellt haben, nur ist der oberste fossile Boden viel deutlicher; abweichend von den letztgenannten Autoren läßt aber Brunnacken auf der A $1 \mathrm{tr}$ i $ß$ - Terrasse (R I) statt richtig JungrißLöß von R II den Altriß-Löß von R I akkumulieren, so daß sein „Altriß/Jungriß“-Interglazial in Wahrheit Soergets R/W, sein „Jungriß"-Löß SoErgeLs W I-Löß, sein „R/W“Boden in Wahrheit Soergels W I/II und sein „W I/II"-Boden SoErgels W II/III-Boden (Paudorf) ist, wie Brunnacker ausdrücklich angibt (1. c. S. 37, 84, 85). Auf der ca. $10 \mathrm{~m}$ tiefer liegenden Terrasse von Spätriß („Jungriß“) = R II stellte BrunNaCKER nur zwei fossile Böden (W I/II und W II/III von SoergeL) im Würm-Löß fest.

5. Ziegelei Arapovichi bei Novgorod-Seversk (NW-Rußland): nach A. A. Velichкo (1961, Fig. 74) liegt an der Desna eine Lößabfolge auf Moräne des Dnjepr (Saale I)-Stadials mit unbedeutender Decke aus dem Odinzowo-Interstadial (Dnjepr/Moskau = Saale I/II): Löß des Moskau (Saale II)-Stadials - fossiler Boden des Mikulino (Eem, Moskaui Valdai $=$ Saale/Weichsel)-Interglazials - Valdai (Weichsel) I-Löß - fossiler Boden Valdai I/II (Weichsel J/II) - Valdai II (Weichsel II)-Löß - fossiler (sehr schwacher) Boden Valdai II/III (Weichsel II/III) - Valdai III (Weichsel III)-Löß - rezenter Boden, also genau wie in Mitteleuropa.

6. Lößprofil von Constanţa am Schwarzen Meer (HAASE \& Richter 1957, Fig. 3 auf S. 167): $2,5 \mathrm{~km}$ nördlich vom Hafen fanden HAASE \& RICHTER ein über $20 \mathrm{~m}$ hohes LößProfil auf „marin-litoral“ gestörtem geringmächtigem Älterem Löß 1-6m über dem Meeresspiegel (nach briefl. Auskunt von Prof. Dr. H. Richter-Leipzig). Diese marine Störung kann, da es die einzige ist, nur durch die Karangat-Transgression im $R / W^{1}$ ) verursacht worden sein. Der Löß über der Störungszone ist also Würm-Löß; er weist vier fossile Bodenbildungskomplexe auf. Im ältesten, dreifachen $4,5 \mathrm{~m}$ mächtigen Komplex $\mathrm{B}_{\mathrm{D}}$ sind nach der Beschreibung der unterste (extremer Rotlehm) der R/W-Boden und die beiden oberen (Lehm bzw. Rotlehm) mit großer Wahrscheinlichkeit die Altwürm-Interstadiale Amersfoort XII und Brörup-Loopstedt wie in dem ähnlichen dreifachen Boden-

1) Im Geograph. Institut in Leipzig wird neuerdings unter Berufung auf (nicht genannte) russische Geologen die Karangat-Transgression ins Mindel/Riß-Interglazial gestellt. Frau Dr. N. S. TscheBOTAREWA vom Geograph. Institut der Akademie d. Wiss. der UdSSR in Moskau erteilte mir die Auskunft (12. 7. 1963), daß niemand in diesem Institut die Richtigkeit der Zuweisung dieser Transgression zum Riß/Würm-Interglazial bezweifle. Von Herrn Dr. Petre Samson vom Institut de Speologie in Bukarest erhielt ich die Auskunft (20. 3. 1964), daß im Moskauer Geolog. Institut W. I. Gromow und seine Schule die Karangat-Transgression ins Mindel/Riß-Interglazial auf Grund einer nachweislich ve rf e hl t e n archäologischen Datierung eingestuft haben (Moustérien vom Beginn des Mindel/Riß-Interglazials bis zum Ende des Riß/Würm-Interglazials, Beginn des Jungpaläolithikums im Anfangsabschnitt von Riß). Ich danke auch an dieser Stelle Frau Dr. N. S. Tschebotarewa und Herrn Dr. Perre Samson verbindlichst für ihre Auskünfte. 
komplex von Unter-Wisternitz in Mähren (KLímA u. Mitarb. 1962); hier wie dort verschmelzen die drei Böden stellenweise zu einem einzigen. Der nächst jüngere Komplex $B_{C}$ ist doppelt und $2 \mathrm{~m}$ mächtig, der nächste $\mathrm{B}_{\mathrm{B}}$ ebenfalls doppelt und der jüngste schwache Boden $\mathrm{B}_{\mathrm{A}}$ nur $0,50 \mathrm{~m}$ mächtig. HA.tSE \& RICHTER weisen mit Recht auf die verblüffende Ahnlichkeit dieses Profils mit niederösterreichischen hin und parallelisieren (vorläufig als Arbeitshypothese) diese Bodenbildungen mit dem Kremser $\left(B_{D}\right)$, dem „Göttweiger“ d. h. Mittelwürm $\left(\mathrm{B}_{\mathrm{C}}\right)$, dem Paudorfer Boden $\left(\mathrm{B}_{\mathrm{B}}\right)$; zum jüngsten Boden $\mathrm{B}_{\mathrm{A}}$ gibt es in Niederösterreich keine Entsprechung, wohl aber in Ungarn (um 18000 B.P.).

7. Die Lößlehmgrube „Biedensteg “ bei Bad Wildungen (E. JaCobshagen \& Mitarb. 1963). Ein ca. 0,70 m mächtiger fossiler Boden trennt zwei Primigenius-Faunen führende würmeiszeitliche Lößlehm-Komplexe (zusammen $6 \mathrm{~m}$ mächtig) in einem Erdfall (Taf. 12); der untere Komplex weist zwei daumendicke EulengewölleSchichten auf (Fig. 1). Die ökologische und paläoklimatologische Auswertung der ungewöhnlich reichen Faunenreste (rund 80 Arten) im unteren Komplex ermöglichte E. JAcoBsHAGEN die Feststellung einer Biotopen-Abfolge (Taf. 14) von einer Steppe mit Waldinseln (offenbar unmittelbar auf das Brörup-Loopstedt-Interstadial folgend) über eine subarktische Steppe zur Tundra, die in einem längeren bodenbildenden Interstadial durch eine Steppe abgelöst wurde (alle Steppennager sind in der gemäßigten kontinentalen Klimazone zuhause!). Erneute starke Abkühlung bewirkte die Fortsetzung der unterbrochenen Ablagerung (zunächst durch Fließerde, dann durch Löß mit hochglazialer Fauna). Der untere Lößlehm-Komplex ist nach den genannten Autoren im älteren, der obere im jüngeren Würm abgelagert worden, d. h. im 3. Stadial von Altwürm bzw. im Jungwürm. Die Steppenphase mit dem fossilen Boden ist also ins Mittelwürm zu stellen.

Für dieses Mittelwürm habe ich bisher den Namen „Göttweiger Interstadia l-Komplex " gebraucht, weil die Göttweiger Bodenbildung seit Soergel von Breuil \& Koslowski, Zeuner, Grahmann, Zotz, Guenther, Brandtner u. a. in diese Periode (als die bekannteste im Löß) gestellt war. Nun hat B. FRENZEL (1964) die Ergebnisse seiner sehr verdienstvollen außerordentlich mühsamen palynologischen Untersuchungen der fossilen Böden des Stillfried A-Komplexes von Oberfellabrunn, des Stillfried B (beide Orte im ariden östlichen Niederösterreich) und der angrenzenden Lößschichten beider Profile veröffentlicht. Diese verschiedenen Schichten lieferten ganz verschiedene Pollenspektra: für die fossilen interstadialen Böden mit größerem Anteil des Baumpollens als in den Lößschichten, in der Verlehmungszone mit besonders viel Pollen letztinterglazialer Holzarten; bei der Ablagerung des Lösses ist der Pollenniederschlag gleichzeitig mit dem Löß abgelagert worden, bei der Bodenbildung ist der Pollenniederschlag in den Interstadialen und Interglazialen in diesen ursprünglich stadialen Löß bei seiner Verwitterung hineingespült worden. Erstaunlich ist, daß auch sehr wenig resistenter Pollen meist in gut erkennbarem Zustand erhalten geblieben ist. Während der Nichtbaumpollen aus der Umgebung der Probenentnahmestelle stammt, kommt beim Baumpollen auch Weit- und Ferntransport in Betracht. Auffällig ist, daß Carpinus-Pollen, der in den letztinterglazialen Pollendiagrammen erst in der zweiten Hälfte auftritt, schon im unverwitterten Rißlöß unter dem letztinterglazialen fossilen Boden erscheint, ferner in diesem auch Fagus, die nördlich der Alpen im Spätpleistozän n icht vorkam, wohl aber in Norditalien und Slovenien, so daß hier unzweifelhaft Ferntransport vorliegt. Der in den verwitternden Rißlöß eingespülte Pollen interglazialer Holzarten mischte sich mit dem stadialen Pollen des Rißlösses, so daß die Verlehmungszone Pollenspektren lieferte, die für eine Vegetation wie die heutige sprechen, aber nicht wie die interglaziale! Trotzdem ist es nach FrenzeL möglich, interstadiale und interglaziale Böden im Löß palynologisch zu unterscheiden, eine außerordentlich wichtige Errungenschaft. Dan ach ist die Verlehmungszone an der Basis des Stillfried A doch letztinterglazial ( $/ W / W$ ), was mit anderen Mitteln nicht zuverlässig bewiesen werden 
k o n n t e, leider auch nicht terrassenmorphologisch. Das Stillfried A von Oberfellabrunn (und sicher auch von Stillfried a. d. March) hat, was BRANDTNER und FINK sowie neuerdings auch tschechische Forscher angenommen haben, die gleiche Zeitstellung wie der erheblich mächtigere dreifache Bodenkomplex von Unter-Wisternitz (KLímA u. Mitarb. 1962), der älter als $55000 \mathrm{C}^{14}$-Jahre ist, während für die oberste Humuszone von Oberfellabrunn nur ein Mindestalter von $42000 \mathrm{C}^{14}$-Jahren gemessen worden war.

Seine Pionierarbeit auf dem Gebiet der Löß-Palynologie plant FREnzeL weiter auszubauen. Dazu gehört außer der palynologischen Untersuchung von fossilen Böden, die auf Grund anderer Befunde zweifellos letztinterglazial sind (z. B. in Unter-Wisternitz), vor allem die palynologische Untersuchung der Verlehmungszone in Göttweig und ähnlicher. Bisher scheint nämlich der Stillf ried A-Komplex mit dem Anfangsabschnitt des Würms nur in besonders ariden Gebieten erhalten geblieben zu sein, aber schon in etwas weniger ariden (z. B. Trockenbecken in Thüringen) sind im Horizont des frühen Altwürms mit seinen beiden Interstadialen nur Schwemmlöß oder Fließerde angetroffen worden (UNGER \& RAU 1961). Sind Verlehmungszonen ohne Auflagerung von je 2 Humusund Löß-Zonen wie in Göttweig auch letztinterglazial? Es gibt zweifellos, wie oben gezeigt wurde, auch mittelwürmzeitliche, also interstadiale Verlehmungszonen, die bisher als Göttweiger bezeichnet worden sind; sind etwa infolge fehlender pedologischer Untersuchung mit modernen Methoden in Niederösterreich und Bayern interstadiale und letztinterglaziale Verlehmungszonen parallelisiert worden? In den Profilen mit dem Stillfried A muß dann noch gefragt werden, wo die mittelwürmzeitliche Verlehmungszone geblieben ist (klimatisch bedingte Lücke im Lößprofil?). Eine Bodenbildung in interstadialen Abschnitten in einer klimatisch sehr unruhigen langen Periode könnte in den kalten Oszillationen \pm abgetragen worden sein; so ist es vielleicht zu erklären, daß in Frankreich die Bodenbildung des Mittelwürms so viel schwächer als im kontinentaleren Osten ist.

$\mathrm{Zu}$ beachten ist in diesem Zusammenhang noch folgendes: BrunNacker (1957, S. 34 Abb. Köfering, S. 38, 73, 86, 90) hat makroskopisch-pedologisch im Regensburger Trokkengebiet festgestellt, daß die beiden vor dem Paudorfer interstadialen Boden (seinem „W I/II"-Boden) gebildeten viel stärkeren fossilen Böden seines „R/W"- und „Altriß/ Jungriß-Interglazials" (in Wahrheit: Mittelwürm W I/II Soengel und R/W Soerget, siehe Abschnitt V, 4) einander sehr ähnlich sind: Pseudogley auf (Para-) Braunerde; der Pedologe Schönhals (in Eiszeitalter u. Gegenwart 1, 1951, S. 109-128) hat auch den fossilen Boden des Würm II/III-Interstadials“ wie den des "Würm I/II-Interstadials" als Braunerde bezeichnet.

Solange die namengebende Verlehmungszone in Göttweig nicht palynologisch (anders geht es nicht!) als mittelwürminterstadial („W I/II") erwiesen ist, darf das Mittelwürm natürlich nicht als „Göttweiger Interstadial“ (F. E. ZEUner 1954) bezeichnet werden, sondern etwa als „Würm-Hauptschwankung“ oder „Würm-Interpleniglazial“, für das, wie oben gezeigt wurde, genügend Beweise vorliegen, so daß an meiner Geochronologie des Spätpleistozäns von 1958 sonst (außer Ergänzungen) nichts zu ändern ist.

\section{Der Klima-Charakter des Mittelwürms}

Im Mittelwürm von (abgerundet) 50000-30000 B.P. stagnierte das Inlandeis innerhalb der Alpen und ebenso im Norden, wie es in England erwiesen ist, mit kleineren und größeren Oszillationen. Diese lange Unterbrechung im (horizontalen) Wachstum des Inlandeises spricht für ein im ganzen interstadiales Klima; daß es im Durchschnitt höchstens boreal (kühl-temperiert) und nur im Süden etwas wärmer war, beweist die Tatsache, daß in NW-England in der Nähe der Inlandeisfront eine von Moränenschutt des zurückschmelzenden (Main) Irish Sea-Gletschers um 42000 B.P. begrabene Toteismasse erst nach 30000 Jahren auftaute, also das Mittelwürm überdauerte (Shotton \& Strachan 1959). 


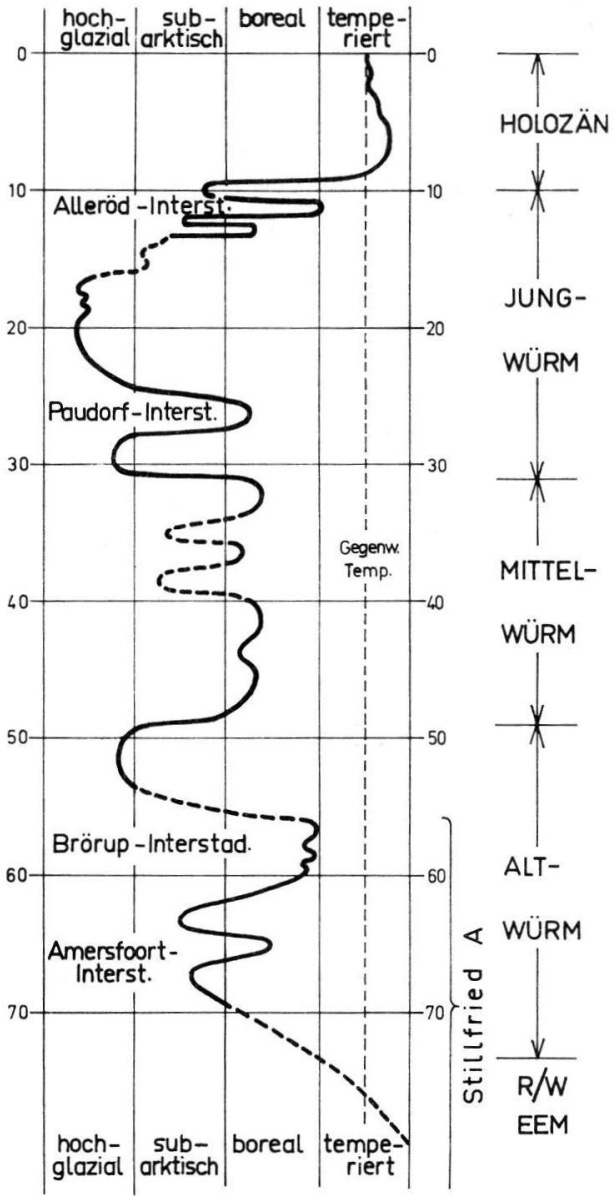

Abb. 1. Schematische Paläotemperatur-Kurve (auf Grund roher Schätzungen der Juli-Mitteltemperatur) der letzten Eiszeit. Die Ci4_ Daten für die beiden Altwürm-Interstadiale sind Mindestdaten; der Beginn der Letzten Eiszeit liegt nach Protaktinium-Daten aus Tiefsee-Bohrkernen etwa 85000 Jahre zurück.

$\mathrm{N}$ amensänderungen: Mittelwürm früher "Göttweiger Interstadial“ (F. E. ZEUNER 1954), „Göttweiger Interstadial-Komplex" (H. Gross) und "großes Würm-Interstadial W I/II" (besonders bei Urgeschichtlern); Jungwürm früher „Hauptwürm“ $(\mathrm{H}$. Gross).

A olische Löß-Ablagerung: In den Kaltphasen v o r dem Brörup-Interstadial $\mathrm{n} u \mathrm{r}$ in den aridesten Klimagebieten, $\mathrm{n}$ a ch dem Brörup-Interstadial a $11 \mathrm{ge}$ $\mathrm{m}$ e in in den Kaltphasen bis zu ihrem hochglazialen Maximum.

Von einem „F-Interglazial“ kann also keine Rede sein; das Mittelwürm war eine lange, aber flache Zäsur in der Temperatur-Kurve der Letzten Eiszeit. Hauptsächlich archäologische Untersuchungen haben paläontologische Befunde ergeben, die für ein unbeständiges Klima sprechen, in dem wärmere und kältere Perioden miteinander abwechselten; es war aber für den Pflanzenwuchs günstig genug, daß er sogar in beträchtlichen Höhen und in der Nähe der Inlandeisfront Großwild ernähren konnte, z. B. in Gebirgen den Höhlenbären, das Charaktertier des Mittelwürms. Das lange kalte 3. Altwürm-Stadial hatte die subarktischen Bäume des Brörup-Loopstedt-Interstadials weit nach $S$ abgedrängt. Das wechselvolle Mittelwürm-Klima verzögerte lange ihre Rückkehr; möglicherweise hat auch das Großwild das Aufkommen von Jungwuchs verhindert (Coope, Shotton \& Strachan (1961). Erst spät erschienen an geschützten Stellen (besonders in Flußtälern) Gruppen von starke Winterkälte ertragenden Holzarten (Pinus, Picea, Larix, Betula u. a.), und im letzten Drittel entstanden in Südfrankreich und im südöstlichen Mitteleuropa taiga-artige Bestände, in denen die Koniferen vorherrschten. Artbestimmungen von Holzkohlen sind für die Paläoklimatologie nur dann verwertbar, wenn sie aus Herden entnommen sind und im Hangenden keine postglazialen Kulturschichten vorkamen, aus denen durch wühlende Tiere z. B. Fagus-Holzkohle ausgerechnet in die Hauptfundschicht der Lemminge verschleppt werden konnte wie in den Weinberghöhlen. Moorbildungen sind für das Mit- 
telwürm bisher nur in den Niederlanden und im nordwestlichen und nördlichen Alpenvorland nachgewiesen worden; anderwärts war sein Klima wahrscheinlich nicht humide genug. Die meisten, wenn nicht alle früher von mir und anderen vermutungsweise ins Mittelwürm gestellten Moorbildungen stammen aus den Altwürm (Altweichsel)-Interstadialen.

\section{Literatur:}

Andersen, Sv. Th., Vries, H1. De, ZAGwijn, W. H.: Climatic change and radiocarbon dating in the Weichselian Glacial of Denmark and the Netherlands. - Geol. en Mijnbouw 39: 38-42, 1960.

BECK, P.: Bericht über die außerordentliche Frühjahrstagung der Schweizerischen geologischen Gesellschaft in Thun 1938. - Eclogae geol. Helvet. 31: 173-198 (speziell 185, 186), Basel 1938.

BrunNacker, K.: Die Geschichte der Böden im jüngeren Pleistozän in Bayern. - Geolog. Bavar. 34 : 5-95, München 1957. - - Bemerkungen zum Profil Hörmating/Obb. - Eiszeitalter u. Gegenwart 13: 125-128, 1962.

Chmielewski, W.: Civilisation de Jerzmanowice. - Inst. Hist. Mat. Polskiej Akad. Nauk, Warszawa 1961, $92 \mathrm{p}$.

Coope, G. R., Shotton, F. W., F. R. S., \& Strachan, I.: A Late Pleistocene flora and fauna from Upton Warren, Worcestershire. - Philos. Transact. Roy. Soc. B, 244: 379-421, London 1961.

Dylik, Jan, Dylikowa, Anna, \& Mitarb.: The Łódź Region. - Guide-Book of Excursion C, VIth INQUA Congress, Eódź 1961.

EBers, Edith: Drumlinkerne, ältere Würmschotter und das Interstadial-Profil von Hörmating/Obb. - Eiszeitalter u. Gegenwart 11: 64-76, 1960.

Frenzel, B.: Zur Pollenanalyse von Lössen (Untersuchungen der Lößprofile Oberfellabrunn und Stillfried, Niederösterreich). - Eiszeitalter u. Gegenw. 15, 1964 (im Druck). - - Ich bin Herrn Dr. Frenzel sehr dankbar dafür, daß er mir im Oktober 1963 ein Duplikat seines Manuskripts zur Kenntnisnahme geliehen hat.

Freund, Gisela: Die Blattspitzen des Paläolithikums in Europa. - Quartär-Bibl. 1, Bonn 1952.

Gross, H.: Das Göttweiger Interstadial, ein zweiter Leithorizont der letzten Vereisung. - Eiszeitalter u. Gegenwart 7: 87-101, 1956. - - Die bisherigen Ergebnisse von Cl4-Messungen und paläontologischen Untersuchungen für die Gliederung und Chronologie des Jungpleistozäns in Mitteleuropa und den Nachbargebieten. - Ebenda 9: 155-187, 1958. - Noch einmal: Riß oder Würm? - Ebenda 10: 65-76, 1959. - - Die Bedeutung des Göttweiger Interstadials im Ablauf der Würm-Eiszeit. - Ebenda 11: 99-106. - - Der gegenwärtige Stand der Geochronologie des Spätpleistozäns in Mittel- und Westeuropa. Quartär 14: 49-68, Bonn 1962-1963. - - Die urgeschichtlichen und geochronoligschen Befunde der Bären- oder Tischofer-Höhle bei Kufstein am Inn. - Quartär 15 (im Druck).

Guenther, E. W.: Zur Altersstellung der paläolithischen Station Murg (Oberrhein). - Jber. u. Mitteil. oberrhein. geol. Ver. 1963.

Guenther, E. W., \& Bronger, A.: Der Fund eines throgontheroiden Elefantenmolaren im Lößprofil von Heitersheim im südlichen Oberrheintal. - Arheološki Vestnik 13/14: 211-222, Ljubljana 1962-1963.

HAase, G., \& Richrer, H.: Fossile Böden im Löß an der Schwarzmeerküste bei Constanţa. Peterm. Geogr. Mitteil. 101: 161-173, 1957.

Jacobshagen, E., Huckriede, R., \& Jacobshagen, V.: Eine Faunenfolge aus dem jungpleistozänen Löß bei Bad Wildungen. - Abh. hess. L.-Amt Bodenforsch. 44, 105 S., 9 Abb., 2 Tab., 14 Taf., Wiesbaden 1963.

Klíma, B., Kukla, J., LožEK, V., † VRIEs, Hl. dE: Stratigraphie des Pleistozäns und Alter des paläolithischen Rastplatzes in der Ziegelei Dolní Věstonice (Unter-Wisternitz). - Anthropozoikum 11 (1961): 93-145, Prag 1962.

Leroi-Gourhan, Arlette: Flores et climat du Paléolithique récent. - C. r. Congrès préhist. de France à Monaco 1959, Le Mans 1960.

Movius, H. L., Jr.: Radiocarbon dates and Upper Palaeolithic archaeology in Central and Western Europe. - Current Anthropology 1: 355-391, Chicago 1960.

Obermaier, H.: Das archäologische Fundmaterial (der Tischofer-Höhle). - In: M. Schlosstr 1910: 484-503. 
PeAke, D. S.: Glacial changes in the Alyn River system and their significance in the glaciology of the North Welsh border. - Quarterly J. geol. Soc. London 117: 335-366 (besonders pp. 361, 362), 1961.

PENCK, A.: Die Vergletscherung der deutschen Alpen. 1882. - - Ablagerungen und Schichtstörungen der letzten Interg]azialzeit in den nördlichen Alpen. - Sber. preuß. Akad. Wiss., phys.math. K1.: 214-251, Berlin 1922.

Penck, A., \& Brǘckner, E.: Die Alpen im Eiszeitalter. Leipzig 1909.

ReIch, Helga: Die Vegetationsentwicklung der Interglaziale von Großweil und Pfefferbichl im Bayerischen Alpenvorland. - Flora 140: 386-443, Jena 1953.

Schlosser, Max, unter Mitwirkung von F. Birkner \& H. Obermaier: Die Bären- oder Tischoferhöhle im Kaisertal bei Kufstein. - Abh. math.-physikal. Kl. d. Kgl. Bayer. Akad. d. Wiss. 24. Bd. (1909): 385-506, München 1910.

Schmid, Elisabeth: Höhlenforschung und Sedimentanalyse. Ein Beitrag zur Datierung des Alpinen Paläolithikums. - Schr. Instit. f. Ur- u. Frühgesch. der Schweiz 13, 185 S., Basel 1958.

Shotron, F. W., F. R. S., \& Strachan, I.: The investigation of a peat moor at Rodbaston, Penkridge, Staffordshire. - Quarterly J, geol. Soc. London 115: 1-16, 1959.

Soerget, W.: Löße, Eiszeiten und paläolithische Kulturen. Jena 1919.

UNGER, K. P., \& RAU, D.: Gliederung und Altersstellung der Lößablagerungen im Thüringer Becken und deren Randgebier. - Instytut Geol. Odbitka z Tomu 34 Prac (INQUA Warszawa 1961): 227-235, Warschau 1961.

VAloch, K.: More on Upper Palaeolithic archaeology, comment. - Current Anthropology 2: 448, Chicago 1961.

Velichro, A. A.: Area of Driepr glaciation (beyond the limits of Moscow glaciation): Relief and stratigraphy of Quaternary deposits. - Relief and stratigraphy of the Quaternary deposits in the northwestern part of the Russian plain: 173-207, Moscow 1961 (russ.).

VÉrtes, L.: Die Höhle von Istállóskő. - Acta Archaeol. Acad. Scient. Hungar. 5: 111-291, Budapest 1955(a). - - Paläolithische Kulturen des Würm I/II-Interstadials in Ungarn. Ebenda 5 (3/4): 261-278, 1955(b). - - Les conditions de l'interstadial würmien I/II hongrois élucidées par l'examen des remplissages des grottes. - Acta Geol. Acad. Scient. Hungar. 3 (4): 395,-407, 1955(c). - - Das Würm-1/2-Interstadial - Wendepunkt des Paläolithikums. - Ber. V. Internat. Kongreß f. Vor- und Frühgesch., Hamburg 1958: 834-838, Berlin 1961. - - Untersuchungen an Höhlensedimenten. Methode u. Ergebnisse. - Régészeti Füzetek Ser. II, 7, 176 S., Budapest 1959.

VRIES, Hl. DE: Radiocarbon dates for upper Eem and Würm-interstadial samples. - Eiszeitalter u. Gegenwart 9: 10-17, 1958. - - Die Chronologie der Würmvereisung. - Ber. V. Internat. Kongreß f. Vor- u. Frühgesch. Hamburg 1958: 840-842, Taf. 52, 3. Berlin 1961.

Wernert, P.: Stratigraphie paléontologique et préhistorique des sédiments quaternaires d'Alsace, Achenheim. - Mém. Service Carte géol. d'Alsace et de Lorraine 14, Strasbourg 1957.

Woldstedt, P.: Uber die Gliederung des Quartärs und Pleistozäns. - Eiszeitalter u. Gegenwart 13: 120-122, 1962.

Wright, H. E., Jr.: Late Pleistocene climate of Europe: a review. - Geol. Soc. Amer. Bull. 72: 933-984, 1961.

ZAGwiJn, W. H.: Vegetation, climate and radiocarbon datings in the Late Pleistocene of the Netherlands. Part I. Eemian and Early Weichselian. - Mem. Geol. Foundat. in the Netherlands, N.S. $14: 15-45,1961$.

Zeuner, F. E.: Riss or Würm? - Eiszeitalter u. Gegenwart 4/5: 98-105, 1954.

Zотz, L. F.: Altsteinzeitkunde Mitteleuropas. Stuttgart 1951.

Zotz, L. F., \& Mitarb.: Das Paläolithikum der Weinberghöhlen bei Mauern. - Quartär-Bibl. 2, 330 S., XV Taf., Bonn 1955.

Manuskr. eingeg. 29. 2. 1964

Anschrift des Verf.: Dr. Hugo Groß, 86 Bamberg, Kunigundendamm 59. 